\title{
Performance of Free Space Optical Communication System with BPSK and QPSK Modulation
}

\author{
Tejbir Singh Hanzra ${ }^{1}$, Gurpartap Singh ${ }^{2}$ \\ ${ }^{1}$ (Department of Electronics and Communication Engineering, Lovely Professional University Jalandhar, India) \\ ${ }^{2}$ (Department of Electronics and Communication Engineering, Lovely Professional University Jalandhar, India)
}

\begin{abstract}
Free Space Optics is the technology which implies the line-of-sight link for the transfer of data between two distant points. The beam of light is used to provide optical connection that can transmit and receive data. The performance of the modulation techniques- Binary Phase Shift Keying (BPSK) and Quadrature Phase Shift Keying (QPSK) are studied in the Nakagami channel and the Rician Channel. The QPSK modulation provides double data rate than the BPSK modulation technique. The certain impairments associated with the FSO system are also studied in this work. The effect of scintillation index and Free Space Path Loss (FSPL) are also considered in this work.
\end{abstract}

Keywords - BER, FSO, FSPL, Nakagami channel, Rician channel, Scintillation.

\section{INTRODUCTION}

Free space optics (FSO) communications gives user, large and unregulated bandwidth. The free space optical communication uses the light signal which carries the information. This light signal is not confined into a physical channel like Optical Fiber. In the Free Space Optical communication the optical signal is transmitted into the free space and the air or vacuum space acts as the channel for signal transmission. The FSO can provide data rate in the range of $100 \mathrm{Gbit} / \mathrm{s}$ and the data transfer is achievable over a distance of $1-4 \mathrm{~km}$. The direct lineof-sight FSO link offer numerous advantages compared to the conventional wired and radio frequency (RF) wireless communications [1].

The main components of an FSO system are modulator, optical source, optical detector and a demodulator. The basic block diagram of a FSO system is given in the Fig. 1.

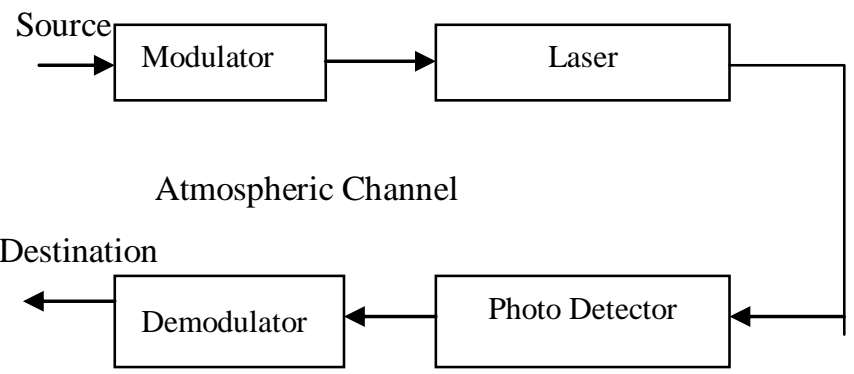

Fig. 1. Basic block diagram of the FSO system

The local area network (LAN) based FSO system has the potential to solve the "last-mile" problem for the foreseeable future as a bandwidth in excess of $2 \mathrm{THz}$ is readily available in optical wavelengths. The performance of the FSO link is hampered by some atmospheric conditions such as- fog, smoke, rain, snow etc. There are few other circumstances when the performance of the FSO system may get affected which include building sway during earthquake or some temporary blockage between line-of-sight connections required for data transmission. The major issue related to this FSO system is the atmospheric turbulence which depends upon the temperature and pressure of the atmosphere [1][2]. The power consumption of FSO link is relatively lower and they also provide greater security to the data as the data carrying light rays are confined to very narrow area as the light beam is of small diameter. The FSO signals are also less sensitive to the electromagnetic interferences.

The modulation and the demodulation both are done in the electrical domain. While the Pulse Position Modulation (PPM) and On-Off Keying (OOK) modulation techniques are used widely, the research is going on to implement other modulation techniques to get the better performance of the system [3]. Free-space point-topoint optical links can be implemented using infrared laser light, although low-data-rate communication over 


\section{Performance of Free Space Optical Communication System with BPSK and QPSK}

Modulation

short distances is possible using LEDs. Infrared Data Association technology is a very simple form of free-space optical communications [4][5].

\section{GAMMA-GAMMA MODELING}

The turbulence model gamma-gamma is based upon such modulation process which assumes that the small scale and large scale effects are responsible for the changes occurring in the path of radiated light signal travelling through turbulent atmosphere. This channel model also considers Fresnel zone which is a region between transmitter and receiver The Fresnel zone is an elliptical area between transmitter and receiver around the line-of-sight path between them. The strongest signal between transmitter and the receiver is carried by this Fresnel zone. The eddies cells which are smaller than the Fresnel zone result in small signal effect i.e. scattering and the eddied cells larger than the Fresnel zone result in large scale effect known as refraction [6].

The refraction may result into complete loss of the information signal. The small scale eddies are represented by $\alpha$ and the large scale eddies are represented by $\beta$. Both these parameters can be related to the atmospheric turbulence as:

$$
\begin{aligned}
& \alpha=\frac{1}{\exp \left[\frac{0.49 \beta_{0}^{2}}{\left(1+1.11 \beta_{0}^{22 / 5}\right)^{7 / 6}}\right]-1} \\
& \beta=\frac{1}{\exp \left[\frac{0.51 \beta_{0}^{2}}{\left(1+1.11 \beta_{0}^{12 / 5}\right)^{5 / 6}}\right]-1}
\end{aligned}
$$

Where, $\beta_{0}^{2}$ is the Rytov variance which depends upon the refractive index, $c_{n}^{2}$.

\section{ISSUES RELATED TO FSO}

The various issues related to the free space optic system are- atmospheric turbulence, scintillation, fog, smoke, building sway etc. These are the parameters which affect the performance of the system. The research is going on to make the FSO system more efficient and accurate.

\subsection{Atmospheric Turbulence}

The atmospheric turbulence is caused by the random fluctuations in the temperature and the pressure of the atmospheric region through which the FSO signal has to pass. This is one those problems which we cannot overcome completely as this depends upon the time of the day as the temperature is majorly controlled by the Sun but still we can improve up to an extent. Due to the temperature changes in the atmosphere the refractive index of the air changes which causes the light beam to deviate from its intended path towards receiver [1][7].

\subsection{Scintillation}

Atmospheric scintillation can be defined as the changes occurring in the light intensities in time and space at the plane of a receiver detecting the optical signal. The received signal at the detector fluctuates because of changes in the refractive index which arise due to change in the temperature of the air along the transmit path. These index changes make the atmosphere to function like a series of small lenses that deflect portions of the light beam into and out of the intended transmit path [7]. The time scale of these fluctuations is in milliseconds, which is approximately equal to the time that it takes a volume of air the size of the beam to move across the path, and therefore is related to the speed of wind to move those lenses like air regions [1]. The effect of scintillation on the optical signal will be highest when the temperature of the day is at the maximum level, this occurs usually during midday. FSO systems operate horizontally in the atmosphere near the surface (in case of terrestrial links), experiencing the maximum scintillation possible.

Scintillation effects for small fluctuations follow a log-normal distribution, characterized by the variance, $\beta_{0}^{2}$ for a plane wave given by the following:

Where $\mathrm{k}=2 \pi / \lambda$

$$
\beta_{0}^{2}=1.23 \cdot \mathrm{c}_{\mathrm{n}}^{2} \cdot \mathrm{k}^{\frac{7}{6}} \cdot L^{\frac{11}{6}}
$$

$c_{n}^{2}$ is the refractive index of the atmosphere which depends upon the temperature and varies throughout the day. Now the equation for scintillation index is as follows: 
$\sigma_{\mathrm{p}}^{2}=\exp \left\{\frac{0.49 \beta_{0}^{2}}{\left(1+0.65 \mathrm{~d}^{2}+1.11 \beta_{0}^{\frac{12}{5}}\right)^{\frac{7}{6}}}+\frac{0.51 \beta_{0}^{2}\left(1+0.69 \beta_{0}^{\frac{12}{5}}\right)^{\frac{-5}{6}}}{\left(1+0.9 \mathrm{~d}^{2}+0.62 \mathrm{~d}^{2} \beta_{0}^{\frac{12}{5}}\right)^{\frac{7}{6}}}\right\}-1$

Here,

$\mathrm{d}=\sqrt{\frac{\mathrm{KD}^{2}}{4 \mathrm{~L}}}$

$\mathrm{D}$ is receiver aperture diameter. From the above equation, scintillation index is calculated and then fading loss can be calculated from the following equation [8]:

$$
\alpha_{\text {scin }}=4.343\left\{1\left\{\operatorname{erf}^{-1}\left(2 \mathrm{P}_{\mathrm{thr}}-1\right) \cdot\left[2 \ln \left(\sigma_{\mathrm{p}}^{2}+1\right)\right]^{\frac{1}{2}}-\quad \ln \left(\sigma_{\mathrm{p}}^{2}+1\right)\right\}\right.
$$

The values of the scintillation index are used to calculate the signal-to-noise ratio (SNR) values which are used later to calculate the bit-error-rate (BER).

\subsection{Free Space Path Loss}

In the field of communication, free-space path loss (FSPL) is the loss in signal strength that would occur in a line-of-sight path through free space (usually air), with no obstacles nearby to cause reflection or diffraction. It does not include factors such as the gain of the antennas used at the transmitter and receiver, nor any loss associated with hardware imperfections [9][10]. The equation for free space path loss (FSPL) and the effect of link length are given below:

$$
\mathrm{FSPL}=\left(\frac{4 \pi \mathrm{d}}{\lambda}\right)^{2}
$$

Where:

$\mathrm{d}=$ distance between transmitter and receiver

$\lambda=$ wavelength.

The wavelength used for the calculation of Free Space Path Loss is $1550 \mathrm{~nm}$.

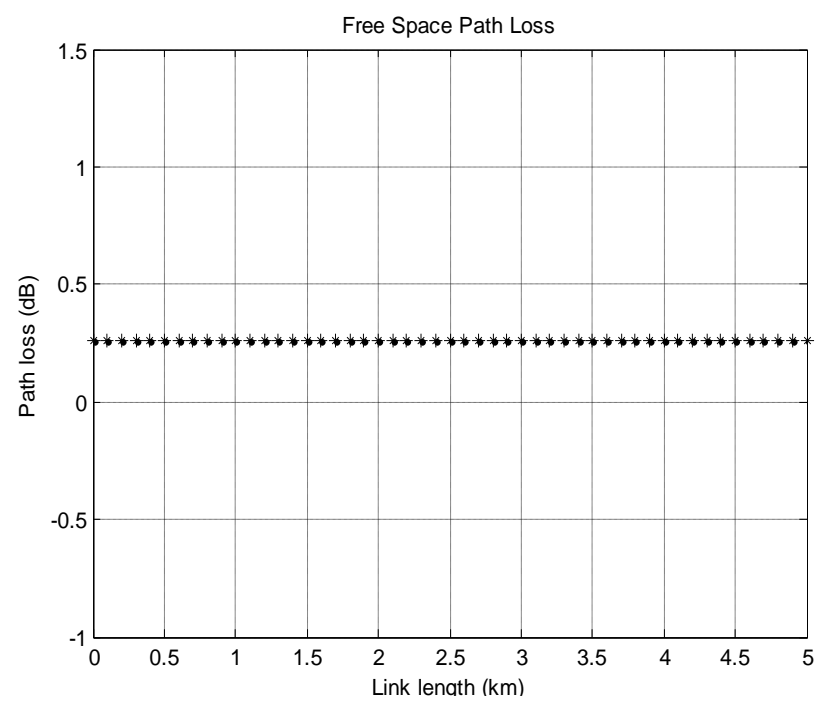

Fig. 2. Free space path loss suffered by FSO signal

The graph given in Fig. 2 shows the FSPL offered by atmosphere to the FSO link which is about $0.26 \mathrm{~dB} / \mathrm{km}$ along the length of link which means that signal will be degraded by $0.26 \mathrm{~dB}$ every $\mathrm{km}$ till the receiver.

\section{MODULATION TECHNIQUES}

There are different options available for the modulation techniques which can be used in the field of Free Space Optical communication such as On-Off Keying (OOK), Binary Phase Shift Keying (BPSK) and QPSK (i.e. Quadrature Phase Shift Keying). The OOK modulation provides good BER performance but data rate is low [5]. The need of the present time is to get high data rate along with good BER performance.

\subsection{Binary Phase Shift Keying (BPSK)}




\section{Performance of Free Space Optical Communication System with BPSK and QPSK \\ Modulation}

Binary Phase-shift keying (BPSK) is a digital modulation scheme that conveys data by changing, or modulating, the phase of a reference signal (the carrier wave). BPSK is appropriate for low-cost passive transmitters and the BPSK is simplest form of phase shift keying (PSK). It uses two phases which are separated by $180^{\circ}$. This modulation is the most robust of all the PSKs since it takes the highest level of noise or distortion to make the demodulator reach an incorrect decision. The BPSK modulation performance is studied in the Nakagami channel and Rician Fading channel. The BER equation of BPSK modulation is as given below:

$$
\mathrm{BER}=\frac{1}{2} \operatorname{erfc}(\sqrt{\mathrm{SNR}})
$$

The Nakagami-m distribution provides the best model to understand land-mobile and indoor mobile multipath propagation as well as scintillating ionospheric radio links. The fading phenomenon in communication channels is due to the presence of time varying multipath. The Nakagami-m distribution has application in the modeling of the path gain of fading channels. Rician fading is a stochastic model for radio propagation anomaly caused by partial cancellation of a radio signal by itself- the signal arrives at the receiver by several different paths (hence exhibiting multipath interference), and at least one of the paths is changing (lengthening or shortening). Rician fading occurs when one of the paths, typically a line of sight signal, is much stronger than the others. In Rician fading, the amplitude gain is characterized by a Rician distribution [11] [12]. The performance of the BPSK modulation in the Nakagami channel is given below in the Fig. 3 .

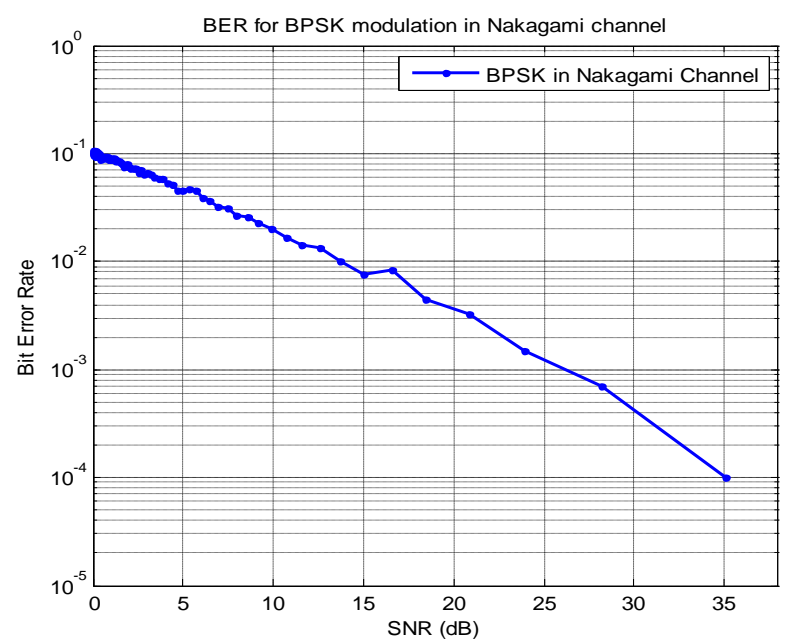

Fig. 3. BER performance of the BPSK modulation in the Nakagami channel

The graph represented in the Fig. 3 depicts the BER performance of the BPSK modulation in the Nakagami channel. The BER value of $10^{-4}$ is achieved at an SNR value of $35 \mathrm{~dB}$. If SNR value is further increased then there are no errors occurring in the received signal which means the BER value becomes zero. The performance of BPSK modulation in the Rician Fading channel is given in the Fig. 4.

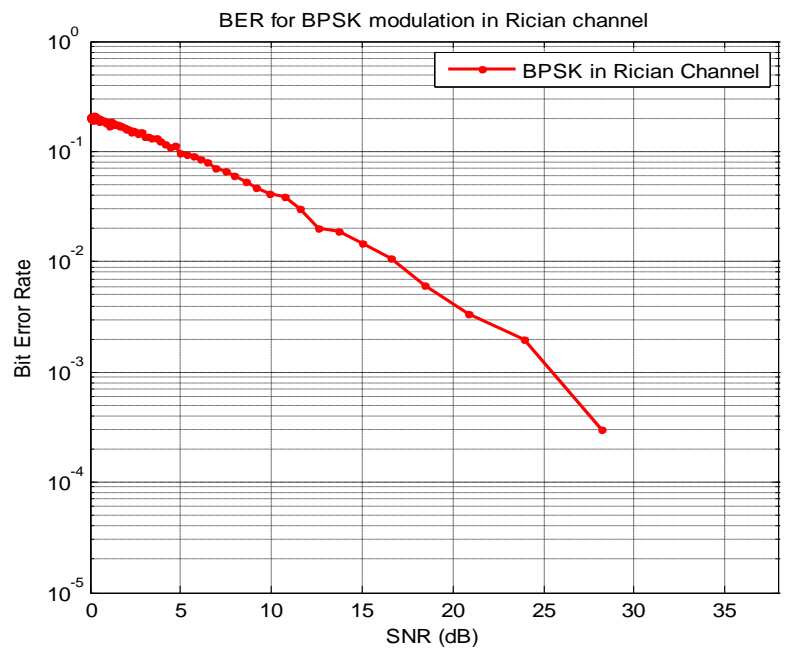

Fig. 4. BER performance of BPSK in Rician Fading Channel 


\section{Performance of Free Space Optical Communication System with BPSK and QPSK \\ Modulation}

The graph represented in the Fig. 4 portrays the BER performance of the BPSK modulation in Rician channel. It is clear as the BER value of $10^{-3}$ is achieved at an SNR value of $26 \mathrm{~dB}$. After the SNR value is increased above $28 \mathrm{~dB}$ then there are no errors occurring in the received signal. The BPSK modulation has a disadvantage of wastage of the bandwidth as one symbol getting transmitted carries only one bit (i.e. 1Bit/symbol).

\subsection{Quadrature Phase Shift Keying (QPSK)}

The Quadrature Phase Shift Keying is one of the variants of PSK modulation which uses four different points on the constellation diagram, equally spaced around a circle to represent the data bits. These four phases help the QPSK to encode two bits per symbol while representing the data [13]. The QPSK is can be used to double the data rate compared with a BPSK system while it maintains the same bandwidth of the signal. The QPSK can also work in a manner, in which it maintains the data-rate of BPSK but make the bandwidth requirement half as compared to BPSK [14]. The bit error rate equation of QPSK modulation is given below:

$$
\mathrm{BER}=\operatorname{erfc}(\sqrt{\mathrm{SNR}})
$$

The performance of the QPSK modulation in the Nakagami channel is given in the Fig. 5. The Nakagami-m distribution often gives the best fit to land-mobile and indoor mobile multipath propagation as well as scintillating ionospheric radio links. The fading phenomenon in communication channels is due to the presence of time varying multipath [10]. Rician fading is a stochastic model for radio propagation anomaly caused by partial cancellation of a radio signal by itself- the signal arrives at the receiver by several different paths (hence exhibiting multipath interference), and at least one of the paths is changing (lengthening or shortening). Rician fading occurs when one of the paths, typically a line of sight signal, is much stronger than the other multipath signal components.

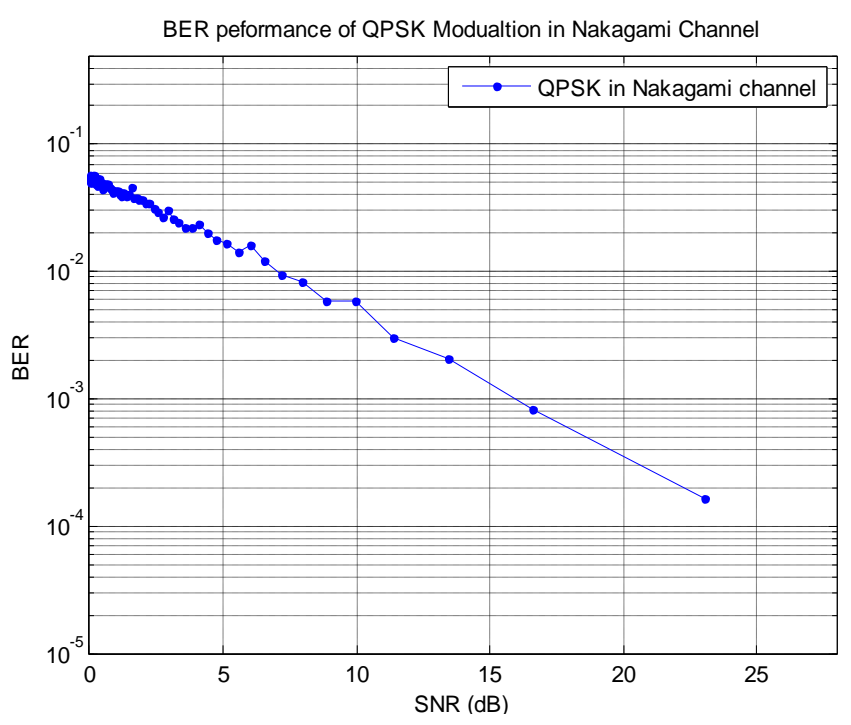

Fig. 5. BER performance of QPSK in the Nakagami channel

The graph given in the Fig. 5 portrays the performance of QPSK modulation in the Nakagami channel. The BER value of $10^{-3}$ is achieved with an SNR of $16 \mathrm{~dB}$ level and if the SNR value is increased above $23 \mathrm{~dB}$ then there is no error received in the signal at the receiver end.

The performance of the QPSK in the Rician channel is given in the Fig. 6. The Rician fading channel considers the presence of one line-of-sight component along with other multipath components arriving at the receiver end. 


\section{Performance of Free Space Optical Communication System with BPSK and QPSK}

Modulation

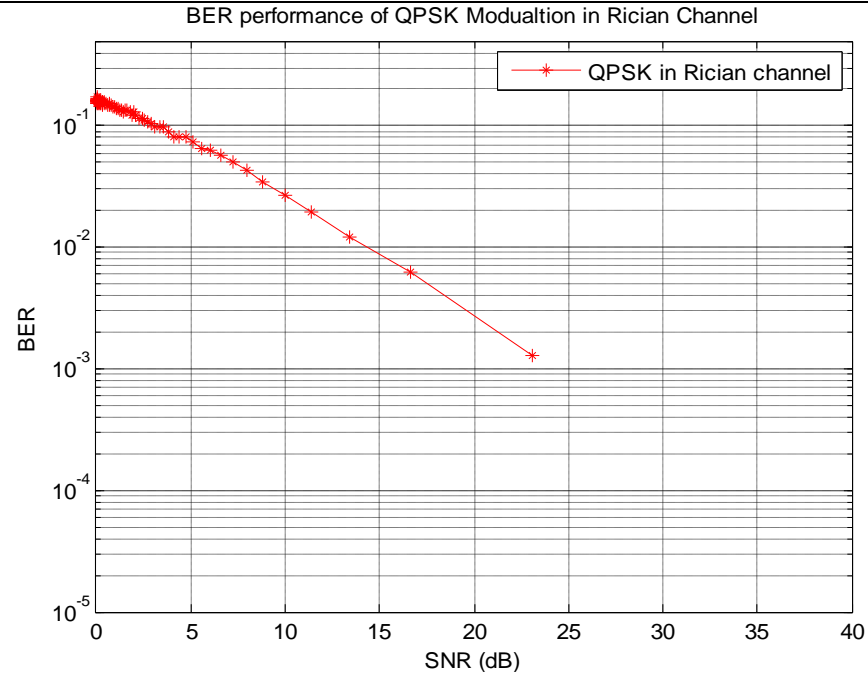

Fig. 6. BER performance of QPSK modulation in the Rician Channel

The graph in the Fig. 6 above portrays the performance of the QPSK modulation as the BER value of $10^{-2}$ is achieved at the SNR value of about $14 \mathrm{~dB}$ and the performance gets further improved as the value of SNR is increased. When the SNR value is increased above $24 \mathrm{~dB}$ the BER value becomes zero.

\section{CONCLUSION}

The Free Space Optics System (FSO) is becoming the solution to the problem of last-mile service providing. The performance of the FSO system utilizing the BPSK and QPSK modulation are studied in the Nakagami channel and Rician channel. The values of the signal to noise ratio (SNR) are calculated by varying the value of scintillation index. The BER value of $10^{-4}$ is can be achieved by the BPSK modulation in the Nakagami channel with an SNR value of $35 \mathrm{~dB}$ and above this value of SNR the BER value becomes zero. In Rician channel the SNR value of $26 \mathrm{~dB}$ is required for achieving $10^{-3}$ value of BER. The BPSK modulation provides lower data rate as compared to QPSK modulation. In the QPSK modulation if the SNR value is $17 \mathrm{~dB}$ then we can achieve $10^{-3}$ value of BER and if the SNR is increased above $24 \mathrm{~dB}$ then BER value becomes zero. In the case of Rician channel if the SNR is increased above $24 \mathrm{~dB}$, the BER value becomes zero. The QPSK modulation provides better performance than the BPSK modulation in terms of data rate. The choice of modulation technique in a particular application will depend on the achieved value of SNR i.e. if the SNR value is more than $20 \mathrm{~dB}$ then the QPSK modulation can be used as it will provide higher data rate for signal transmission. The OOK modulation used currently can be replaced by the QPSK modulation to get higher data rate and higher bandwidth efficiency if we can achieve the SNR value above 20dB.

\section{REFERENCES}

[1] S. Bloom, E. Korevaar, J. Schuster, and H. Willebrand, "Understanding the performance of free-space optics", Journal of Optical Networking, Optical Society of America, Vol. 2, Issue 6, 2003, 178-200.

[2] C. Motlagh, V. Ahmadi, Z. Ghassemlooy and K. Abedi, "The Effect of Atmospheric Turbulence on the Performance of the Free Space Optical Communications", $6^{\text {th }}$ IEEE International Symposium on Communication Systems, Networks and Digital Signal Processing, 2008, 540-543.

[3] D. Kedar and S. Arnon, "Urban optical wireless communication networks", IEEE Communications Magazine, 2004, s2-s7.

[4] F. Xu, M.A. Khalighi and S. Bourennane, Ecole Centrale Marseile, Institut Fresnel, "Pulse Position Modulation for FSO Systems: Capacity and Channel Coding", $10^{\text {th }}$ IEEE International Conference on Telecommunication, ConTEL, 2009, 31-38.

[5] B. Barua, "Comparison the Performance of Free-Space Optical Communication with OOK and BPSK Modulation under Atmospheric Turbulence", International Journal of Engineering Science and Technology (IJEST), Vol 3, No. 5, 2011, 4391-4399.

[6] Z. Wang, W.D. Zhong, S. Fu and C. Lin, "Performance Comparison of Different Modulation Formats Over Free-Space Optical (FSO) Turbulence Links with Space Diversity Reception Technique", IEEE Photonics Journal, Volume 1, Number 6, 2009, 277285.

[7] Z. Ghassemlooy and W.O. Popoola, "Terrestrial Free Space Optical Communication", Mobile and wireless communications: Network layer and Circuit level design, 17 (2010) 355-391.

[8] P.T. Dat, A. Bekkali, K. Kazaura, K. Wakamori and M. Matsumoto, "A Universal Platform for Ubiquitous Wireless Communications Using Radio Over FSO System", Journal of Lightwave Technology, Volume 28, Number 16, 2010, 2258 - 2267.

[9] S.S. Muhammad, E. Leitgeb and O. Koudelkat, "Multilevel Modulation and Channel Codes for Terrestrial FSO links", 2nd IEEE International Symposium on Wireless Communication Systems, 2005, 795-799.

[10] O. Bouchet and H. Sizun, Free-Space Optics Propagation and Communication (Chippenham, Wiltshire, Antony Rowe Ltd, 2006).

[11] G.S. Prabhu and P.M. Shankar, "Simulation of Fading using Matlab for Classroom Instruction".

[12] T.D. Rappaport, Wireless Communication Principles and Practice (Second edition, New Delhi, Pearson Education India, 2009).

[13] F. Xiong, Digital Modulation Techniques (Second edition, Norwood, MA, Artech House, Inc., 2006).

[14] S.G. Wilson, Digital Modulation and Coding (New Jersey, Prentice Hall, 1996). 